\title{
Designing Medical CBIR System with Edge Density Criterion
}

\author{
Kulwinder Singh Mann and Er. Jagbir Singh Gill
}

\begin{abstract}
The Medical Image can be understood by its visual features also known as image signatures. These features when modified or enhanced under certain geometrical functions, it improves the resultant retrieval by the increase of 94\% in the performance with the Comparison of $85 \%$ in the case of the nearest neighbor and in the case of normal image Retrieval it comes out to be $75 \%$. This Research includes the approach of calculating edge density is applicable for the five human body parts- Hands, Pelvis, Breast, Brain and Chest and is cam parable to the system in the previous work in literatures. The Result acquired for the proposed system comprises of edge density concepts.
\end{abstract}

Index Terms-Medical images, edge density, geometric feature vector, content based image retrieval.

\section{INTRODUCTION}

Content-based image retrieval has its deep roots in medical organization in such a way that it is regarded as best way to retrieve information from medical images that comes out as output of the system .Medical images has become backbone of medical organization giving growth to the new field known as medical imaging diagnosis [1]. Accepting the challenge of the access to the more relevant images for the users give the options of easy and improve search of the image [2]. Generally, CBIR system working approaches include the drawing out of feature vectors that also named as image signature [3].and its comparison with the similarities of the visual query image so that relevance features and same proportion can be achieved. Also, neural networks are the modern trends of CBIR adopted methods for latest and fastest techniques for retrieval with very less time consuming. Generally, CBIR get categorized into two classes when user involvement is concerned. One is relevance feedback for the active learning strategies and other is processing of image databases through different classification [4]. This Research work involves second task which has been enhanced in the classification, comparison and in the retrieval of advanced images features. The proposed research is designed into different sections as section [5]

\section{PREVIOUS Related WorK}

Medical Images have become vital resources in organizing and searching of many treatments and diseases

Manuscript received December 10, 2014; revised January 30, 2015.

The authors are with Guru Nanak Dev Engineering College, Ludhiana, India (e-mail:mannkulvinder@yahoo.com, gill@gmail.com). through the content based image Retrieval system [6]. Extracting the features of the medical images has approaches differently for medical images . Characterization of the features is admittance in the system by Andoloussi et al. [2] by the Bi-dimensional Empirical Mode Decomposition with generalized density function. Feature Vectors are determined for the feature extended version of extraction and can be based on any feature of image like color in [3] by discrete cosine transform method. Feature shape is in consideration for the system like Redial Basis Functions with Relevance feedback .

\section{WORKFLOW OF THE SYSTEM}

The Research work has proposed system with an idea from [7]. the diagram shows the particular flow of the process from point the query image to the finally retrieved image. The system flow can be broadly categorized as: 1) Database and extraction-system has one general database that stores the medical images of every format in it, and system's other database stores the feature vectors extracted from the system during the process of Extraction. 2) Image Retrieval- It is the last comparison phase finished with the categorization of the image.

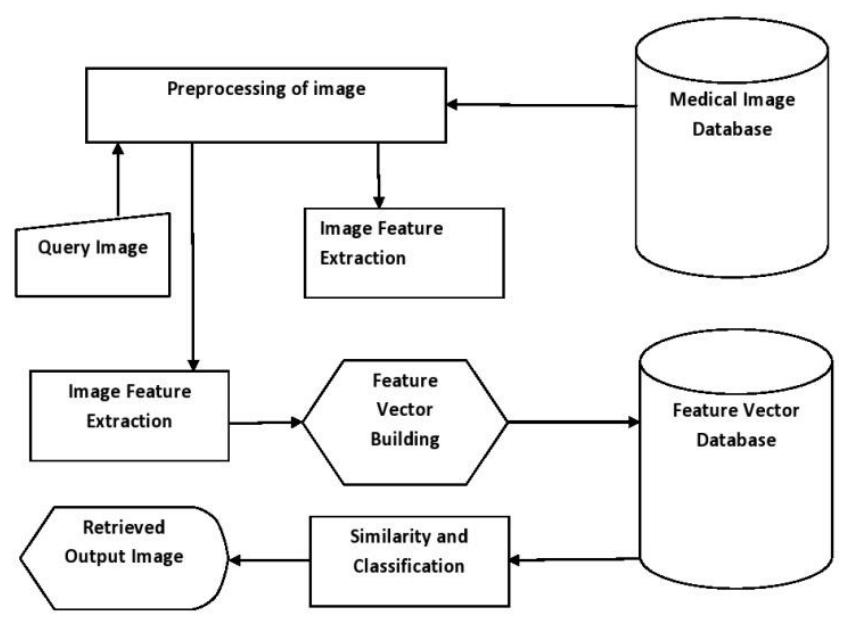

Fig. 1. Content based image retrieval system.

The system shown in Fig. 1 has been designed to retrieve the image most relevant to the query image. Its working can be elaborated into two phases as:

\section{A. Preprocessing and Extraction Phase}

Database having number of Medical images along with them noisy data different formats redundant information. Whole the proposed process work on specified format for which all images has to redefine in accordance to the system 
required image format [8]. Preprocessing is the procedure to alter the existing images to formulate a new image by formatting it this is done for avoiding the unrealistic data involved in the image.

\section{B. Reforming the Image}

Major aspect for the process of reforming the medical image is to make the image clearer for the system so that it can have sharp visible edges and the noisy data can be eliminated. Laplace filter operator has been performing for two dimensional images in the system shown in [9].

$$
D^{L}=D_{m}^{L}+D_{n}^{L}=\left[\begin{array}{ccc}
0 & 1 & 0 \\
1 & -4 & 1 \\
0 & 1 & 0
\end{array}\right]
$$

Next Prior task to the enhancement, is converting the image into the binary format. Grayscale image pixels are computed for their threshold values to calculate the spread of each class of variance given in (2). Using threshold variance it is given in (4). After certifying the image formation from only white and black pixels the image is ready for the feature extraction phase[10].

$$
\alpha^{2}=f_{1} \alpha_{1}^{2}+f_{2} \alpha_{2}^{2}
$$

In general it can be given as

$$
\begin{array}{r}
f_{x}=\sum_{a}^{b} p(i) \\
\alpha^{2}=f_{1} f_{2}\left(\mu_{1}-\mu_{2}\right)^{2}
\end{array}
$$

And here $\alpha$ is the spread of each class of variance Known as Otsu value, $f_{x}$ is the weighting of the class with Probability $p(i)$ of the class and $\mu$ is the interclass variance.

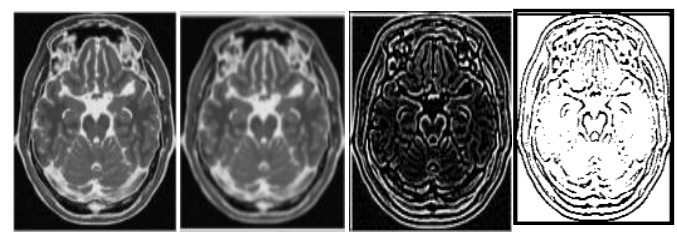

Fig. 2. The actual Image is shown in 2(a) is the original image and 2(b), 2(c) are the after the adaptive threshold figure $2(d)$ is the binary image.

\section{Division of Images by Geometrical Functions}

The Functionality of the System for the extracting the features of the medical images is two geometrical parameters[11]. One is the rectangular and other is circular parameter namely Rectangular Segments and circular radii respectively[12]. In each parameter point of the images are taken in small rectangle or in circle to draw a window or circle around the image as many times as it can cover the whole image.

\section{1) Rectangular segments}

Division of images into Segments are started from the left side of the image the vertical and horizontal points of the images are defined by $\left(x_{0}, y_{0}\right)$ and $\left(x_{1}, y_{1}\right)$. Moving with these points the 7 segments of local edge feature of the image and eighth is global image are made shown in figure $3(\mathrm{a})-3(\mathrm{~h})$ and the points defined on the extreme left elevated $L_{e}$ and extreme right lowered $R_{l}$ with width $w$ and height $h$ of the image can be given under each segment from (5) - (12) below:-

$$
\begin{array}{rlrl}
L_{e} 0 & =(0,0), & R_{1} 0=(w, h) \\
L_{e} 1=(0,0), & R_{1} 1=\left(\frac{w}{2}, \frac{h}{2}\right) \\
L_{e} 2=\left(0, \frac{h}{2}\right), & R_{1} 2=\left(\frac{w}{2}, h\right) \\
L_{e} 3=\left(\frac{w}{2}, 0\right), & R_{1} 3=\left(w, \frac{h}{2}\right) \\
L_{e} 4=\left(\frac{w}{2}, \frac{h}{2}\right), & R_{1} 4=(w, h) \\
L_{e} 5=\left(\frac{w}{4}, \frac{h}{4}\right), & R_{1} 5=\left(\frac{3 w}{4}, \frac{3 h}{4}\right) \\
L_{e} 6=\left(0, \frac{h}{4}\right), & R_{1} 6=\left(\frac{w}{2}, \frac{3 h}{4}\right) \\
L_{e} 7=\left(\frac{w}{2}, \frac{h}{4}\right), & R_{1} 7=\left(w, \frac{3 h}{4}\right)
\end{array}
$$

\section{2) Circular radii}

Division of images into segments is started from the centre of the image to increasing radius of the circle moving outwards of the images. Each circular region produced by varying radius of the image will contain different feature of the same image. There will be five Circular regions obtained for one single image. Each circular region here is given by $C_{r}$, with maximum value $C_{(\text {rmax })}$ with height $H_{c}$ and width $W_{c}$ of the image with radius

Of centre $\mathrm{R}$ with $\left(R, c_{o}\right)$ extracted edges. so, $C_{(\max )}=$ $\max \left\{H_{c}, W_{c}\right\}$. They can be shown as

$$
\begin{gathered}
C_{r} 0=1 \\
C_{r} 1=C_{r} 0+(R) \\
C_{r} 2=C_{r} 0+2(R) \\
C_{r} 3=C_{r} 0+3(R) \\
C_{r} 4=C_{r} 0+4(R) \\
C_{r} 5=C_{r} 0+5(R)
\end{gathered}
$$

Each region represented in (13) -(18) has its pictorial presentation in figure with general expression as:

$$
\text { - } C_{r} n=C_{r} 0+n(R) \ldots
$$

and

$$
R=\frac{C_{r_{n}}-C_{r_{0}}}{n-1}
$$
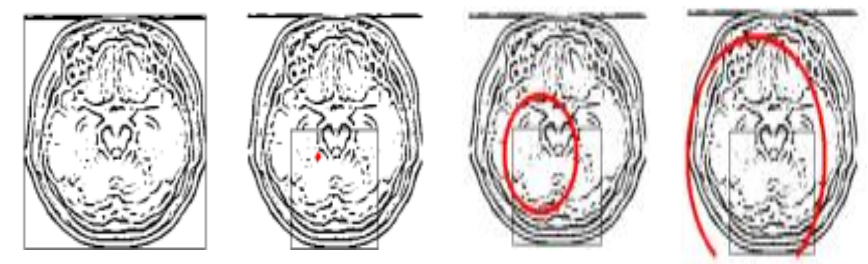

Fig. 3. Shows the Medical image with the feature vector with circular radii.

The Images shown is the Fig. 3 is the feature vector collection with geometrical Radii. The medical images are 
of varying sizes so the geometrical parameters adjust the center of the images by composing the virtual height and width of the images. Fig. 5 shows the image of chest with different size with full circle.

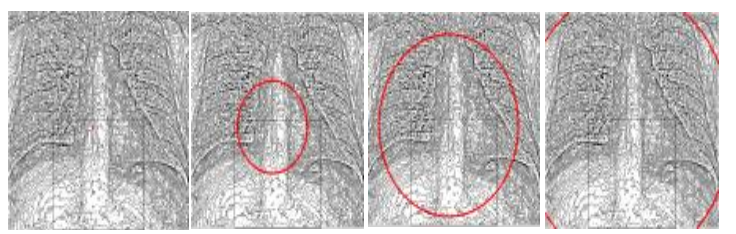

Fig. 4. Shows the image with circular radii with feature vector

\section{Computing Edge Density and Feature Vectors}

The features created in each segment or region has collection of edges from different parts of images. The total calculated part of each region is given Edge density[13]. In the case of rectangular segment edge density is denoted by $\rho$ in (21),including $R_{a}$ and $E_{r}$ where $R_{a}$ is the area and edge magnitude is given by $E_{r}$.

$$
\begin{aligned}
& \rho=\frac{1}{R_{a}} \sum_{m=x_{0}}^{x_{1}} \sum_{n=y_{0}}^{y_{1}} E_{r}(m, n) \\
& R_{a}=\left(x_{1}-x_{0}+1\right)\left(y_{1}-y_{0}+1\right)
\end{aligned}
$$

Compromising total eight feature vector of these segments are:

$V_{r 1}, V_{r 2}, V_{r 3}, V_{r 4}, V_{r 5}, V_{r 6}, V_{r 7} V_{r 8}$. And in the case of the circular radii it is given in the form of pseudo code as:-

For $a=1$ to $a=n-1$

Num $=1$;

For $b=1$ to $b=R$

if $\sqrt{\left(R_{b}-\frac{W_{c}}{2}\right)^{2}+\left(c_{0}-\frac{H_{c}}{2}\right)^{2}}>C_{r 0}$ and

$\sqrt{\left(R_{b}-\frac{W_{c}}{2}\right)^{2}+\left(c_{j}-\frac{H_{c}}{2}\right)^{2}}<C_{r 0} a+1$.

Num=Num +1 ;

Else

Num=Num+0;

End if;

$F\{a\}=$ Num;

END For;

The Five vectors are these segments are $V_{c 1}, V_{c 2}, V_{c 3}, V_{c 4}, V_{c 5}$ Giving the total features of 13 features. The total thirteen features of each image are taken in consideration before going to next phase. Hence, improving the retrieval process of the image from ordinary retrieval system of medical image [14].

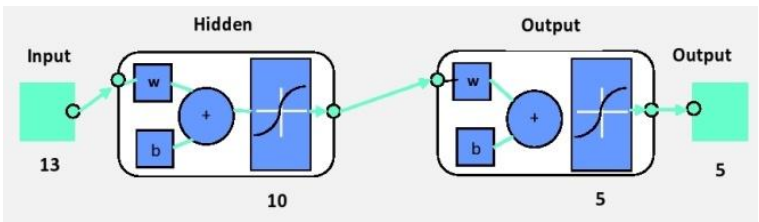

Fig. 5. Outflow of the neural network.

The tested images are repossess from the analysis of the Euclidean distance among all the trained and tested images and forming the category of the image resulting classification. Parameter over which classification is done is the Five nearest neighbor resulting a category which holds five relevant images that are much closer to the query images. Thus retrieving the image among the cluster which has least distance from the similarity features of query image is finally retrieved as an output against the query image.

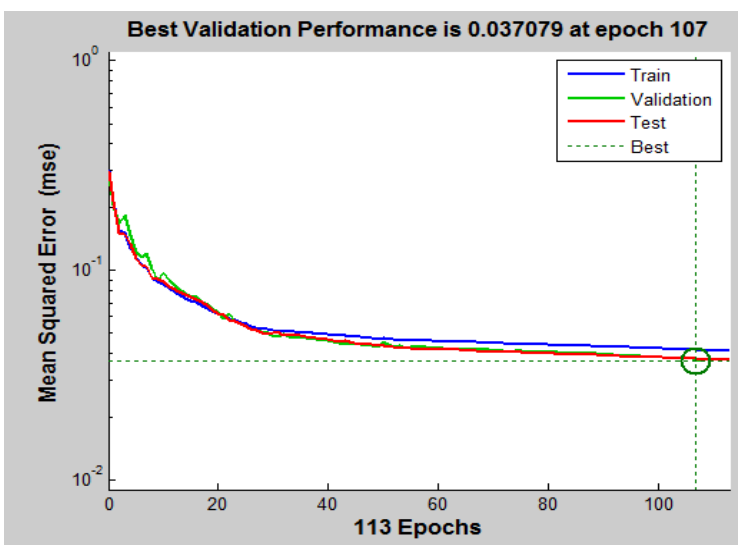

Fig. 6. Performance graph for the system during training.

\section{RESUlTS AND DISCUSSION}

Understanding an image to retrieve the right output is the most important aspect of the proposed system. It access to the testing of the performance of the system and its comparison checks are done by the total thirteen new feature vector in addition of the basic image signature of the image. The importance of this extraction can be seen in the performance of the system that has been used to retrieve the image using these extracted features. The performance can be shown through the graphs of the retrieval image and confusion matrix. Calculated Precision is shown as:

$$
P=\frac{\text { no.of applicable images Retrieved }}{\text { total no.of images retved }}
$$

TABLE I: SHOWING THE RETRIEVAL RATE WITH ITS TwO FEATURE

\begin{tabular}{|l|l|l|l|l|l|}
\multicolumn{5}{c|}{ EXTRACTION FUNCTIONS } \\
\hline \multirow{2}{*}{$\begin{array}{l}\text { Query } \\
\text { Image }\end{array}$} & \multicolumn{5}{|c|}{ Databases } \\
\cline { 2 - 6 } & Hands & Pelvis & Breast & Skulls & chest \\
\cline { 2 - 6 } & \multicolumn{5}{|c|}{ Precision Rate of Retrieving image } \\
\hline 1 & 90 & 70 & 70 & 100 & 80 \\
\hline 2 & 100 & 60 & 80 & 100 & 70 \\
\hline 3 & 100 & 90 & 80 & 100 & 70 \\
\hline 4 & 100 & 80 & 90 & 100 & 80 \\
\hline 5 & 100 & 80 & 100 & 100 & 80 \\
\hline
\end{tabular}

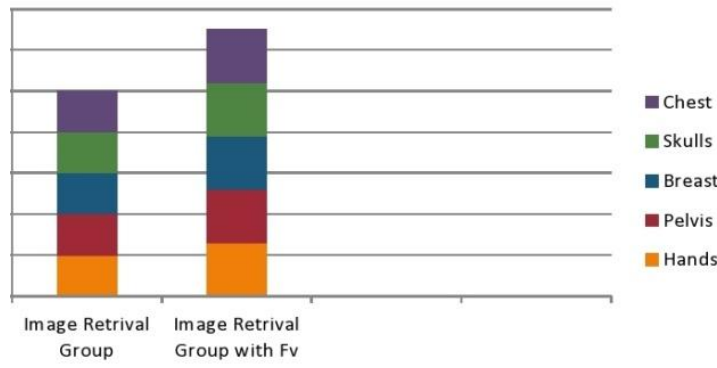

Fig. 7. Showing the result for the two different body parts group.

where is the precision rate in (23). The tabular form of the precision rate during the retrieval of the medical images is shown in Table 1.closer Analysis of the tables shows that System works on the five types of the X-rays of parts of Human body namely Hands, Pelvis, Breast, Skulls and Chest. For each part there have been five Query images for the precision rate of 25 images at the end of the Run. 
Similarly this is done for four more runs [15]. The collaborated precision for all four runs and comparison with the five nearest neighbor for 125 images are given in Table II with distinguished feature vector for retrieval system with its combined two geometric parameters and the graphical notation for the same is given in Fig. 8-Fig. 9. Overall testing performance is shown in the form of confusion matrix in Fig. 8.

TABLE II: COLLABORATED RESULT OF ALL RUNS WITH DIFFERENT QUERY IMAGES

\begin{tabular}{|c|c|c|c|}
\hline Run No & $\begin{array}{c}\text { 5-NN Precision } \\
(\%)\end{array}$ & $\begin{array}{c}\text { Image Retrieval } \\
\text { Precision (\%) }\end{array}$ & $\begin{array}{c}\text { ImageRetrieval } \\
\text { with Fv vector }\end{array}$ \\
\hline 1 & 80 & 80 & 97 \\
\hline 2 & 80 & 61 & 90 \\
\hline 3 & 90 & 86 & 95 \\
\hline 4 & 90 & 76 & 94 \\
\hline 5 & 88 & 80 & 98 \\
\hline
\end{tabular}

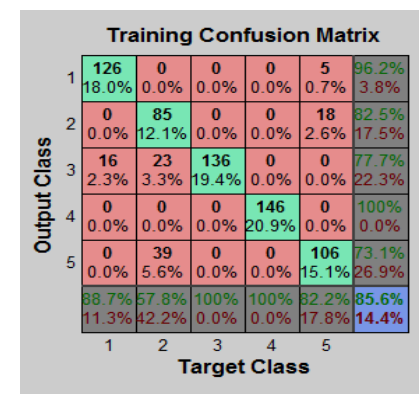

Test Confusion Matrix

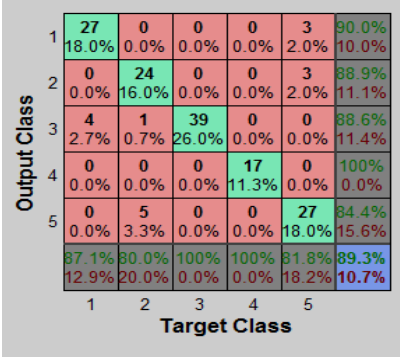

Fig. 8. Confusion matrixes showing the result for the retrieval system.

\section{CONCLUSION}

The research work have been structured on the basis of features of the medical image which are analyzed under geometrical functions with the performance of $76.6 \%$, $85.6 \%, 94.8 \%$ for image retrieval, five nearest neighbor and retrieval with feature vectors. Edge density here with new developed feature vector has proven its impact in the improve precision which has been working well in the considerably previous work of Image Retrieval. The classification and the new features related to the basic parameters of the image can be improved or developed respectively.

\section{REFERENCES}

[1] S. Akbarpour, "A review on content based image retrieval in medical diagnosis," International Journal on Technical and Physical Problems of Engineering, vol. 5, pp. 148-153, 2013.

[2] E. Abdelijail, C. Abdelmajid, M. Nabil, and S. Adderralim, "Medical content based image retrieval by using the HADOOP framework," in
Proc. the $20^{\text {th }}$ International Conference on Telecommunication, 2013, pp. 1-6.

[3] B. Vibha and P. B. Sandeep, "CBIR using DCT for feature vector generation," International Journal Application or Innovations in Engineering and Management, pp. 196-200, 2012.

[4] H. S. Chavan and S. S. Deepak, "Content based image retrieval: Review," International Journal of Emerging Technology and Advanced Engineering, vol. 2, no. 9, 2012.

[5] C. Yixin, W. James, K. Robert, Content Based Image Retrieval by Clustering, MIR'03, Berkley, California, U.S.A, 2003.

[6] C. John and O. Kazunori, "Learning metrics for content based medical image retrieval," in Proc. the $30^{\text {th }}$ Annual International Conference of the IEEE EMBS, 2013, pp. 3363-3366.

[7] T. Dharni and I. A. Laurence, A Survey on Content Based Image Retrieval, 2002.

[8] D. L. Rafael, B. Renato, and R. X. Marcel, "Reducing the complexity of K-nearest diverse neighbor queries in medical image datasets through fractal analysis," in Proc. the $26^{\text {th }}$ International Symposium on Computer-based Medical System, 2013, pp. 101-106.

[9] D. Ritendra, L. Jai, and W. Z. James, "Content Based Image Retrieval -Approaches and Trends of the New age," in Proc. the 7th ACM SIGMM International Workshop on Multimedia Information Retrieval, 2005, pp. 253-264.

[10] E. John and G. Margaret, "Content based image retrieval," JISC Technology Applications, vol. 39, 1999.

[11] E. John and G. Margaret, "Content based image retrieval," Tech Rep.JTAP-039,JISC Technology Application Program, Newcastle upon Tyne, 2000.

[12] F. Michael, K. Ashnil, K. Jinman, and F. Dagan, "Graph based retrieval of PET-CT images using vector space embedding," in Proc. the $26^{\text {th }}$ International Symposium on Computer-based Medical System, 2013, . 413-416.

[13] T. P. Fund and S. M. Marchand, "Dynamic multimedia annotation tools," in Proc. the SPIE Photonics West Conference Proceedings, Internet Imaging III San José, California, USA, 2002, vol. 4672, pp. 216-224.

[14] K. S. Nenad, C. K. Slobodan, Z. J. Goran, and R. D. Branimir, "Implementation of neural network in CBIR system with relevance feedback," Journal of Automatic Control University of Belgrade, pp. 41-44, 2006.

[15] G. Amira and M. Kirmene, "A novel Approach of content based medical images indexing system based on spatial distribution of vector descriptors," in Proc. the $10^{\text {th }}$ International Multi Conference on systems, Signals and Devices(SSD), 2013, pp. 1-4.

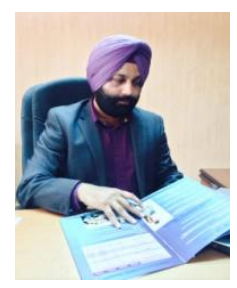

Kulwinder Singh Mann received his dynamic professional and Ph.D. he has more than fifteen years of experience conducting high level academic research. he is a proactive leader and team player integral to success of numerous research initiatives and he focused on dedicated scholar with strong teaching experience and strategic approach to problem solving. he has a resourceful and versatile work ethic, and reputation for integrity with excellent interpersonal skills, highly developed

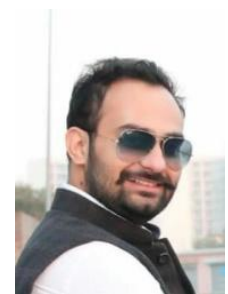

Er. Jagbir Singh Gill received his masters in computer science and engineering. He has experience of four years in the field of medical informatics and pursing his Ph.D. in the field of content based image retrieval. 